\title{
A Class of Separation Axioms in Generalized Topology
}

\author{
D+ ANABALAN AP ${ }^{1}$ AND SANTHI C ${ }^{2}$
}

${ }^{1}$ Head, Department of Mathematics, Alagappa Govt Arts College Karaikudi

Tamilnadu

${ }^{2}$ Research Scholar, Alagappa Govt Arts College Karaikudi Tamilnadu

Received: September 15, 2015| Revised: September 27, 2015| Accepted: February 11, 2016

Published online: March 30, 2016

The Author(s) 2016. This article is published with open access at www.chitkara.edu.in/publications

\begin{abstract}
The purpose of this paper is to introduce and study some new class of definitions like $\mu$-point closure and $g_{\mu}$-regular space concerning generalized topological space. We obtain some characterizations and several properties of such definitions. This paper takes some investigations on generalized topological spaces with $g_{\mu}$-closed sets and $\mathrm{g} \mu$-closed sets.
\end{abstract}

Keywords: $\mu$-compact, $g_{\mu}$-closed set, $g \mu$-closed set, $g_{\mu}^{*}$-closed, a $\mu T_{D^{-}}$ space, $g_{\mu}$-regular space.

\section{INTRODUCTION}

This paper is concerned with the adaptation of the change of topology approach from topological topics to aspects of the theory of generalized topological spaces. This shows that "the change of generalized topology" exhibits some characteristic analogous to change of topology in the topological category. A general application of the change of generalized topology approach occurs when the spaces are ordinary topological spaces. In this case, the generalized topologies are families of distinguished subsets of a topological space which are not topologies but are generalized topologies. Some common examples of generalized topologies that are associated with a given topological space. Consider the collection of all s.o, p.o, $\beta$-open, $\alpha$-open sets in the (ordinary) topology $(X, \tau)$. Each collection is a generalized topology on $X$. In fact, the family of $\alpha$-open set is a topology. But in general, the other three collections, namely, the family of s.o, p.o and $\beta$-open sets are not topologies on $X$.

In 1992, Blumberg defined what he meant by a real-valued function on Euclidean space being densely approached at a point in its domain. Continuous functions satisfy his condition at each point of their domains. Since then, and particularly in the past four decade, a large number of

Mathematical Journal of Interdisciplinary Sciences Vol. 4, No. 2, March 2016 pp. 151-159 
DKanabalan, AP Santhi, C

properties closely related to the notion of continuity of a function have been introduced. The number of properties so large that different authors have used the same term for different concepts and other authors have resorted to exotic terms, some times because the natural term has already been preempty. It turns out that many of these concepts are not new in the sense that if one is willing to change the topology on the domain and /or the range then the class of functions satisfying a particular property often coincides with the class of continuous functions under the new topologies from their point of view many of the results in the literature concerning such functions are essentially restatements in disguise of familiar properties of continuous functions. The main purpose of our paper is to make this more precise in generalized topology.

In this paper we continue our study in the style of [5]. Section 2 is devoted to preliminaries and section 3 is devoted to a brief review of the $\mu$-compact and $g_{\mu}$-regular spaces and some new results are derived. The properties with which we shall be dealing in this paper are quite diverse and include among others, the property of being a zero set, or a $G_{\delta}$-set, being a(regularly) closed set, being a point closure, being a connected set or a compact set, being a strongly regular closed set etcetera.

Intensive research on the field of generalized topological space $(X, \mu)$ was done in the past ten years as the theory was developed by A.Csaszar[1], A.P.Dhana Balan[5]. For background material, paper[5] may be perused.

\section{PRELIMINARIES}

Let $X$ be a set. A subset $\mu$ of $\exp X$ is called a generalized topology on $X$ and $(X, \mu)$ is called a generalized topological spaces [1] (abbr.GTS) if $\mu$ has the following properties:

(i) $\varphi \in \mu$,

(ii) Any union of elements of $\mu$ belongs to $\mu$.

A generalized topology $\mu$ is said to be strong [2] if $X \in \mu$. For the space $(X, \mu)$, the elements of $\mu$ are called $\mu$-open sets and the complement of $\mu$-open sets are called $\mu$-closed sets. For $A \subset X$, we denote by $C_{\mu}(A)$ the intersection of all $\mu$-closed sets containing $A$, that is the smallest $\mu$-closed set containing $A$, and by $i_{\mu}(A)$, the union of all $\mu$-open sets contained in $A$, that is the largest $\mu$-open set contained in $A$. It is easy to observe that $\mathrm{c}_{\mu}$ and $\mathrm{i}_{\mu}$ are idempotent and monotonic, where $\gamma: \exp X \rightarrow \exp X$ is said to idempotent if and only if $A \subset B \subset X$ implies $\gamma(\gamma(A))=\gamma(A)$ and monotonic if and only if $A \subset B \subset X$ implies $\gamma(A) \subset \gamma(B)$. It is also well known that from [3,4] that if $\mu$ is a generalized 
topology on $X$ and $A \subset X, x \in X$ then $x \in \mathrm{c}_{\mu}(A)$ if and only if $x \in M \in \mu \Rightarrow M \cap A \neq \varphi$ and $c_{\mu}(X-A)=X-i_{\mu}(A)$.

Definition 2.1 A subset A of $(X, \mu)$ is

(i) $\mu$-semiopen in $X$ if $A \subset c_{\mu}\left(i_{\mu}(\mathrm{A})\right)$.

(ii) $\mu$-preopen if $A \subset i_{\mu}\left(c_{\mu}(\mathrm{A})\right)$.

(iii) $\mu$ - $\beta$-open if $A \subset c_{\mu}\left(i_{\mu}\left(c_{\mu}(\mathrm{A})\right)\right)$.

(iv) $\mu$ - $\alpha$-open if $A \subset i_{\mu}\left(c_{\mu}\left(i_{\mu}(\mathrm{A})\right)\right)$.

(v) $\mu$-regular open if $A=i_{\mu}\left(c_{\mu}(\mathrm{A})\right)$. The complement of $\mu$-semi open, $\mu$ - $\alpha$ open sets are $\mu$-semi closed, $\mu$ - $\alpha$ - closed.

Definition 2.2 A GTS $(X, \mu)$ is said to be

(i) $\mu$-Hausdorff [11] if for any two distinct points $x$ and $y$ in $X$,there exists disjoint $\mu$-open sets $\mathrm{U}$ and $\mathrm{V}$ such that $x \in \mathrm{U}, y \in \mathrm{V}$.

(ii) $\mu$-regular [11] if for each $\mu$-closed set $\mathrm{F}$ and each point $x \notin \mathrm{F}$, there exists disjoint $\mu$-open sets $\mathrm{U}$ and $\mathrm{V}$ such that $x \in \mathrm{U}, \mathrm{F} \subseteq \mathrm{V}$.

\section{3. $\propto$ - COMPACT AND $g_{\propto}$ - REGULAR SPACES}

Definition 3.1 A subset $A$ of $X$ is said to be a point closure if $A$ is the $\mu$-closure of a singleton. In particular, $A$ is said to be a $\mu$-point closure if it is the $\mu$-closure of a $\mu$-open singleton set. A strong generalized topological space (SGT) $X$ is called a $\mu T_{D}$ - space if $x \in X$, then there are $\mu$-open set $U$ and $\mu$-closed set $F$ such that $U \cap F=\{x\}$.

\section{Example 3.1}

(i) One point compactification is $\mu$-point closure.

(ii) Every closed interval is the $\mu$-point closure of its $\mu$-s.o interval.

(iii) $X=\{\mathrm{a}, \mathrm{b}, \mathrm{c}\}, \mu=\{\phi,\{\mathrm{a}\},\{\mathrm{b}\},\{\mathrm{a}, \mathrm{b}\}\}, \mu$-closed $=\{X,\{\mathrm{~b}, \mathrm{c}\},\{\mathrm{a}, \mathrm{c}\},\{\mathrm{c}\}\}$.

Let $\mathrm{A}=\{\mathrm{a}, \mathrm{c}\}$. Then $\mathrm{A}$ is a $\mu$-point closure of $\{\mathrm{a}\}$.

Result: Every $\mu$-point closure is $\mu$-closure.

Theorem 3.1: Let $X$ be a space and $x \in X$. Let $A \subset X$ be $\mu$-point closure. Then $A$ $=X-\cup\left\{\mu-\mu_{x}\right\}$ where $\mu_{x}=\{U: x \in U \in \mu\}$.

Proof: Since $A$ is $\mu$-point closure, we have $A=c_{\mu}(\{x\})$ for some $x \in X$. Let $y \in c_{\mu}$ $(\{x\})=X-i_{\mu}(X-\{x\})$. Then $y \notin i_{\mu}(X-\{x\})$. Suppose $U \in \mu_{y}$, then $U \not \subset X-\{x\}$, Hence, $x \in U$. That is $U \in \mu_{x}$ So, $\mu_{y} \subset \mu_{x}$ and hence $\mu-\mu_{x} \subset \mu-\mu_{y}$ It follows that $\cup\left(\mu-\mu_{x}\right)$ $\subset \cup\left(\mu-\mu_{y}\right)$. This implies that $y \notin \cup\left(\mu-\mu_{y}\right)$ and so $y \notin \cup\left(\mu-\mu_{x}\right)$. Consequently,
A Class of Separation Axioms in Generalized

Topology 
DKanabalan, AP Santhi, C

$y \in X-\cup\left(\mu-\mu_{x}\right)$. On the other hand, let $y \in \cup\left(\mu-\mu_{x}\right)$. Then we have $y \in \mathrm{c}_{\mu}(\{x\})$ by reversely the proof above. This proves that $\mathrm{c}_{\mu}(\{x\})=X-\cup\left(\mu-\mu_{x}\right)=A$.

Definition 3.2: Let $X$ be a space (i) Let $x \in X$ and $U \in \mu$. Then $x$ is called a representative element of $U$ if $U \subset V$ for each $V \in \mu_{x}$. (ii) A space $X$ is called a $c_{o}$-spaceif $c_{o}=X$, where $c_{o}$ is the set of all representative element of sets of $\mu$. Result: Let $A$ and $B$ be subsets of a $c_{o}$-space. Then (i) $i_{\mu}(A \cap B)=i_{\mu}(A) \cap i_{\mu}$ (B).

(ii) $c_{\mu}(A \cup B)=c_{\mu}(A) \cup c_{\mu}(B)$.

Theorem 3.2: Let $X$ be a $\mu T_{D}, c_{o}$-space and let $A$ and $B$ be $\mu$ - open and $\mu$ closed sets respectively.

Then $i_{\mu}(A \cap B)=i_{\mu}(\{x\})$.

Proof: Let $A \subset X$ be $\mu$-open. Since $X$ is $\mu T_{D}$, there exists $\mu$-closed set $B$ in $X$ such that $A \cap B=\{x\}$. As $X$ is a $c_{o}$-space, $i_{\mu}(A \cap B)=i_{\mu}(A) \cap i_{\mu}(B)=i_{\mu}(\{x\})$.

Definition 3.3: Let $X$ be a SGT space and let $A \subset X$. Then (i) $A$ is said to be a $\mu T_{2}$ - closed set relative to $X$ iff every open cover $\mathcal{A}$ of $A$ has a finite subfamily $\mathcal{A}^{\prime} \subset A$ such that $A \subset \cup\left\{c_{\mu}(\mathrm{U}) / \mathrm{U} \subset \mathcal{A}^{\prime}\right\}$.

(ii) $A$ is said to be $\mu T_{2}$-closed set iff $(A, \mu / A)$ is $\mu T_{2}$-closed.

Example 3.2: Let $(X, \mu)=(X, \tau)$ be a SGT space with the indiscrete topology. Every subset of $X$ is $\mu$-open and $\mu$-dense in $X$. So for every open cover $\mathcal{A}$ and $\mathrm{U} \in \mathcal{A}, c_{\mu}(U)=X$ and $(\mathrm{X}, \mu)$ is $\mu T_{2}$ - closed.

Definition 3.4: A space $X$ is said to be locally $\mu T_{2}$-closed if for each $x \in X$ and a $\mu$-open set $U$ containing $x$, the $\mu$-closure of $U$ is $\mu T_{2}$-closed.

Theorem 3.3: Let $X$ be a SGT space. Then (i) if $A \subset X$ is $\mu T_{2}$-closed set relative to $X$, then $A$ is $\mu$-closed in $X$ if $X$ is $\mu$-Hausdorff. (ii) if $A \subset X$ is $\mu$-open set, $A$ is $\mu T_{2}$-closed set relative to $X$ iff $A$ is $\mu T_{2}$-closed set.

Proof: (i) Let $x \in X$-A. Since $X$ is a $\mu$-Hausdorff space, there exists for each $y \in A, \mu$-open neighbourhoods $U_{y}$ and $V_{y}$ of $\mathrm{x}$ and $y$ respectively such that $U_{y} \cap$ $V_{y}=\phi$. Then $\left\{V_{y} / y \in A\right\}$ is a $\mu$-open cover, hence a $\mu$-open cover of $A$. Since A is $\mu T_{2}$-closed, there exist a finite subset $B \subset A$ such that $A \subset \cup\left\{c_{\mu}\left(V_{y}\right) / y \in B\right\}$. Let $U=\left\{U_{y} / y \in B\right\}$. Then $U$ is a $\mu$-open neighbourhood of $x$ such that $A \cap U=$ $\phi$. Then $c_{\mu}(A)=A$ and hence $A$ is $\mu$-closed.

(ii) Assume $\mathrm{A}$ to be a $\mu T_{2}$-closed set. Then $(A, \mu / A)$ is $\mu T_{2}$-closed. Let $\left\{U_{y}\right\}$ $y \in A\}$ be a $\mu$-open cover of A with $U_{y} \in \mu$-open set of $X$ for every $y \in A$. Let $V_{y}$ $=A \cap U_{y}$. Since $A$ is $\mu$-open, $V_{y} \in \mu$-open set of A. So $\left\{V_{y} / y \in A\right\}$ is a $\mu$-open cover of $\mathrm{A}$ in $\mathrm{A}$. Since $(\mathrm{A}, \mu / \mathrm{A})$ is $\mu T_{2}$-closed, there exists a finite subset $B \subset A$ such that $A \subset \cup\left\{\left(V_{y}\right) / y \in B \subset A\right\}$ (or) $A \subset \cup\left\{\mu-c l_{A}\left(V_{y}\right) / y \in B \subset A\right\}$. Now $\mu-c l_{A}\left(V_{y}\right)=c_{\mu}\left(V_{y}\right) \cap A \subset c_{\mu}\left(V_{y}\right)$. So $A \subset \cup\left\{c_{\mu}\left(U_{y}\right) / y \in B \subset A\right\}$. 
Thus A is $\mu T_{2}$-closed set relative to $X$. Since $A$ is $\mu$-open, every $\mu$-open subsets of $A$ is $\mu$-open in $X$, and hence the converse part of (ii) is obvious.

Definition 3.5: [10] A GTS $(X, \mu)$ is $\mu$-compact if every $\mu$-open cover of $X$ has a finite subcover. A subset $A$ of $X$ is said to be $\mu$-compact relative to $(X, \mu)$ if every cover of A by $\mu$-open sets of $\mathrm{X}$ has a finite subcover.

Definition 3.6: A collection $\mathcal{A}$ of subsets of a SGT space $X$ is said to be a $\mu$-covering of $X$ if the union of the elements of $\mathcal{A}$ equal to $X$. It is called a $\mu$-open covering of $X$ if its elements are $\mu$-open subsets of $X$.

Definition 3.7: A space $X$ is said to be $\mu$-compact if every $\mu$-open covering $\mathcal{A}$ of $X$ contains a finite sub collection that also covers $X$.

Example 3.3: The subspace $X=\{0\} \cup\left\{\frac{1}{n} / n \in Z_{+}\right\}$of $\quad$ is $\mu$-compact. Given a $\mu$-open covering $\mathcal{A}$ of $X$, there is an element $U$ of $\mathcal{A}$ containing 0 . The set $U$ contains all but finitely many of the points $\frac{1}{n}$; choose, for each point of $X$ not in $U$, an element of $\mathcal{A}$ containing it. The collection consisting of these elements of $\mathcal{A}$, along with the element $U$, is a finite subcollection of $\mathcal{A}$ that covers $X$. Let $Y$ be a subspace of $X$. A collection of subsets of $X$ is said to be a covering of $Y$ if the union of it elements contains $Y$.

Theorem 3.4: Let $Y$ be a subspace of $X$. Then $Y$ is $\mu$-compact iff every covering of $Y$ by $\mu$-open sets in $X$ contains a finite sub collection covering $Y$.

Proof: Suppose $Y$ is $\mu$-compact. Let $A=\left\{A_{\alpha}\right\}_{\alpha \in J}$ be a covering of $Y$ by $\mu$-open sets in $X$. Since $Y$ is a subspace of $X,\left\{A_{\alpha} \cap Y / \alpha \in J\right\}$ is $\mu$-open in $Y$. Then the collection $\left\{A_{\alpha} \cap Y / \alpha \in J\right\}$ is a covering of $\mathrm{Y}$ by $\mu$-open sets in $\mathrm{Y}$. Hence $\left\{A_{\alpha 1}, A_{\alpha 2}, \ldots, A_{\alpha n} \cap Y\right\}$ covers Y. Then $\left\{A_{\alpha 1}, A_{\alpha 2}, \ldots, A_{\alpha n}\right\}$ is a sub collection of $\mathcal{A}$ that covers $Y$. Conversely, suppose that the given condition holds. Let $A^{\prime}=\left\{A_{\alpha}^{\prime}\right\}$ be a covering of $Y$ by $\mu$-open sets in $X$. By hypothesis, some finite sub collection $\left\{A_{\alpha_{1}}, A_{\alpha_{2}}, \ldots, A_{\alpha_{n}}\right\}$ covers Y. Then $\left\{A_{\alpha_{1}}^{\prime}, A_{\alpha_{2}}^{\prime}, \ldots, A_{\alpha_{n}}^{\prime}\right\}$ is a subcollection of $\mathcal{A}^{\prime}$ that covers $\mathrm{Y}$, and so $\mathrm{Y}$ is $\mu$-compact.

Theorem 3.5: Every $\mu$-closed subset of a $\mu$-compact space is $\mu$-compact.

Proof: Let $\mathrm{F}$ be a $\mu$-closed subset of a $\mu$-compact space $\mathrm{K}$ and let $\left\{V_{\alpha}\right\}$ be a covering of $F$ by sets $\mu$-open in $X$. Let $F^{c}=\mathrm{W}$. Then $\mathrm{W}$ is $\mu$-open (single $\mu$-open set) form an $\mu$-open covering of $\mathrm{K}$ by adjoining $\left\{V_{\alpha}\right\}$ with $F^{c}$. Then $W \cup\left({ }_{\alpha}^{\cup} V_{\alpha}\right)$ covers $\mathrm{K}$. This $\mu$-open cover of $\mathrm{K}$ contains a finite (sub cover) sub collection $\left\{V_{\alpha_{i}}\right\}$ such that $K \subset W \cup V_{\alpha_{1}} \cup V_{\alpha_{2}} \cup \ldots \cup V_{\alpha_{n}}$. Suppose this sub collection contains the set $\mathrm{W}$, then discard $\mathrm{W}$. If this sub collection does not
A Class of Separation Axioms in Generalized

Topology 
DKanabalan, AP Santhi, C

contain the set $\mathrm{W}$, then leave the sub collection alone. The resulting collection is a finite sub collection of $\mathrm{F}$. Then $F \subset V_{\alpha_{1}} \cup \ldots \cup V_{\alpha_{n}}$.

Theorem 3.6: Suppose $X$ is a $\mu$-Hausdorff space, $\mathrm{K} \subset X, \mathrm{~K}$ is $\mu$-compact, and $\mathrm{p} \in$ $F^{c}$. Then there are $\mu$-open sets $\mathrm{U}$ and $\mathrm{W}$ such that $\mathrm{p} \in \mathrm{U}, \mathrm{K} \subset \mathrm{W}$ and $\mathrm{U} \cap \mathrm{W}=\phi$ Proof: Given $X$ is a $\mu$-Hausdorff space, $\mathrm{K} \subset \mathrm{X}$ is $\mu$-compact and $\mathrm{q} \in \mathrm{K}$. Since $\mathrm{X}$ is $\mu$-Hausdorff and $\mathrm{K} \subset \mathrm{X}$, we have, there exist disjoint $\mu$-open sets $U_{q}$ and $V_{q}$ such that $\mathrm{p} \in U_{q}$ and $\mathrm{q} \in V_{q}$.

Since $\mathrm{K}$ is $\mu$-compact, the collection $\left\{V_{q} / q \in \mathrm{K}\right\}$ is a covering of $\mathrm{K}$. Then there are points $q_{1}, q_{2}, \ldots, q_{n} \in \mathrm{K}$, there exist finite sub collection $V_{q_{1}} \cup V_{q_{2}} \cup \ldots \cup V_{q_{n}}$ such that $\mathrm{K} \subset V_{q_{1}} \cup V_{q_{2}} \cup \ldots \cup V_{q_{n}}$. Then the $\mu$-open set $\mathrm{W}=V_{q_{1}} \cup V_{q_{2}} \cup \ldots \cup V_{q_{n}}$. contains $\mathrm{K}$ and it is disjoint from the $\mu$-open set $\mathrm{U}=U_{q_{1}}^{q_{1}} \cup U_{q_{2}}^{q_{2}} \cup \ldots \cup U_{q_{n}}$ formed by taking the intersection of the corresponding $\mu$-open sets of $\mathrm{p}$. Therefore $\mathrm{U}$ is a $\mu$-open set of $\mathrm{p}, \mathrm{K} \subset \mathrm{W}$ and $\mathrm{U} \cap \mathrm{W}=\phi$

Corollary 3.7: (a) $\mu$-compact subset of a $\mu$-Hausdorff spaces are $\mu$-closed. (b) If $\mathrm{F}$ is $\mu$-closed and $\mathrm{K}$ is $\mu$-compact in a $\mu$-Hausdorff space, then, $\mathrm{F} \cap \mathrm{K}$ is $\mu$-compact.

Proof: (b) follows from (a) and Theorem 3.5

Theorem 3.8: The image of a $\mu$-compact space under a $\mu$-continuous map is $\mu$-compact.

Proof: Let $X$ be $\mu$-compact. and let $f: X \rightarrow Y$ be $\mu$-continuous. Let $\mathcal{A}$ be a covering of the set $f(x)$ by $\mu$-open sets in Y. The collection $\left\{f^{-1}(A) / A \in \mathcal{A}\right.$ \} is a collection of sets covering $X$. These sets are $\mu$-open in $X$ because $f$ is $\mu$-continuous. Hence finitely many of them $f^{-1}\left(A_{1}\right), \ldots, f^{-1}\left(A_{n}\right)$,(say) cover $X$. Then the sets $A_{1}, \ldots, A_{n}$ cover $f(x)$. This implies that $f(x)$ is $\mu$-compact.

Theorem 3.9: Let $f: X \rightarrow Y$ be a bijective $\mu$-continuous function. If $\mathrm{X}$ is $\mu$-compact and $\mathrm{Y}$ is $\mu$ - Hausdorff then $f$ is a homeomorphism.

Proof: The $\mu$-continuity of the map $f^{-1}$ follows if the images of $\mu$-closed sets of $X$ under $f$ are $\mu$ - closed in $Y$. Suppose that $A$ is $\mu$-closed in $X$. Then by Theorem $3.5, A$ is $\mu$-compact. Thus, by Theorem $3.8, f(A)$ is compact. Since $\mu$-compact subset of a $\mu$-Hausdorff space is $\mu$-closed, we have $f(A)$ is $\mu$-closed in Y.

Remark: If $X$ and $Y$ are GTS and $f: X \rightarrow Y$, we call $f$ an $\mu$-open ( $\mu$-closed) set $A$ in $X, f(A)$ is $\mu$-open iff $\mathrm{f}$ is $\mu$-closed iff $f^{-1}$ is continuous. Thus a 1-1 onto map $f$ is a homeomorphism iff it is $\mu$-continuous and $\mu$-open iff it is $\mu$-continuous and $\mu$-closed.

If $(X, \mu)$ is a GTS, then we say that a subset $A \in \delta \subset \rho(X)$ is a $\delta$-set[4] if for every $x \in A$, there exists a $\mu$-closed set $\mathcal{A}$ such that $x \in i_{\mu}(\mathcal{A}) \subset A$. Then $(X, \delta)$ is a GTS, [[4] proposition 2.1] suchthat $\delta \subset \mu[4]$ Theorem1] Elements of $\delta$ 
are called $\delta$-open sets of $(X, \mu)$, For $A \subset X . i_{\delta}(A)$ and $c_{\delta}(A)$ are respectively the interior and closure of $A$ in $(X, \delta)$. The family of all $\alpha$-open (resp. semi open, pre open, b-open, $\beta$-open) sets of the generalized topological spaces $(X, \delta)$ denoted by (resp. $\xi, \eta, \varepsilon, \psi$ ).

Definition 3.8: [8] Let $(X, \mu)$ be a generalized space. A subset $A$ of $X$ is said to be $g_{\mu}$-closed if $c_{\mu}(A) \subset M$ whenever $A \subset M$ and $M \in \mu$. Various properties of $g_{\mu}$-closed sets are discussed and characterizations are given in[2] and these properties are valid for the generalized topologies induced by $\mu$ and $\delta$. Given a topological space $(X, \tau)$ and a generalized topology $\mu$ on $X$, a subset $A$ of $X$ is said to be $\mathrm{g} \mu$-closed if $\mathrm{c}_{\mu}(A) \subset M$ whenever $A \subset M$ and $M \in \tau$. If $\mathrm{M}=\mathrm{I}$, then $\mathrm{g}$ -closed sets coincide with the $\mathrm{g}$-closed sets of Levine[7] [ie, $A \subset X$ is $\mathrm{g}$-closed if $\operatorname{cl}(A) \subset U$ whenever $A \subset U$ and $U$ is open in $X]$.

The difference between the two definitions $\mathrm{g}_{\mu}$-closed set and $\mathrm{g} \mu$-closed set is that the definition of $g \mu$-closed sets uses elements of the topology $\tau$ on $X$ where $X \in \tau$ where as the definition of $\mathrm{g}_{\mu}$-closed sets uses elements of the generalized topology $\mu$ where $X$ may or may not be in $\mu$. Therefore, the definition of $g_{\mu}$-closed sets is more general, since the definition uses a large class of generalized topologies which also contains the class of all topological spaces. Moreover, similar results established for $g \mu$-closed sets are already established for $\mathrm{g}_{\mu}$-closed sets in [5]. The following definition is the definition for generalized closed sets in generalized spaces.

Definition 3.9: A subset $\mathrm{A}$ of $M_{\mu}=\cup\{B / B \in \mu\}$ of a generalized spaces $(X, \mu)$ is said to be $g_{\mu}^{*}$ - closed if $c_{\mu}(A) \cap M_{\mu}$ whenever $A \subset M$ and $M \in \mu$.

Note that, if the space is strong, then this definition coincides with the definition of $\mathrm{g}_{\mu}$-closed sets. The above definition is the common definition of generalized closed sets in generalized spaces for both strong and non strong spaces.

Note that $M_{\mu}=\cup\{A / A \in \mu\}$ and $X \notin \mu$.

Examples: Let $(X, \mu)$ be a GT. Suppose $M_{\mu}=\cup\{A / A \in \mu\} \neq X$ and $\tau=\rho\left(M_{\mu}\right) \cup\{X\}$. Then every $\mu$-closed subset of $X$ contains $X-M_{\mu}$. Therefore, every subset A of $M_{\mu}$ is neither a $g_{\mu}$-closed set nor a $\mathrm{g} \mu$-closed set. Also $g_{\mu}^{*}$-closed sets depend on the generalized topology $\mu$. Every non-empty subset $\mathrm{B}$ of $X$ such that $\mathrm{B} \cap\left(X-M_{\mu}\right) \neq \phi$ or $\mathrm{B} \subset\left(X-M_{\mu}\right)$ is not contained in any $\mu$-open set which implies that such sets are trivially $g_{\mu}$-closed set. Clearly, such sets are $\mathrm{g} \mu$-closed set, since $X$ is the only open set containing such sets.

Definition[3]: Let $(X, \tau)$ be a topological spaces and $\mu$ be a generalized topology on $X$. $(X, \tau)$ is said to be a $\mu g$-regular spaces if for each closed set $F$ and a point $x \notin F$, there exist disjoint $\mu$-open sets $U$ and $V$ such that $x \in U, F \subset V$.
A Class of Separation Axioms in Generalized

Topology

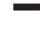


DKanabalan, AP Santhi, C

Example 3.4: Let $X=\{a, b, c\}$ and let $\mu=\{\phi\{a\},\{b\},\{a, b\}\}$ be the family of all generalized open sets, which is not strong. Consider the set $\{a\}$ and $\{b\}$. Then $c_{\mu}(\{\mathrm{a}\})=\{\mathrm{a}, \mathrm{c}\}$ and $c_{\mu}(\{\mathrm{b}\})=\{\mathrm{b}, \mathrm{c}\}$. It is easy to show that $\mu$ is not $\mu g$ -regular.

Example 3.5: Let $\left.X=I_{n}=\{1,2, \ldots, \mathrm{n}\}.\right\}$, Define $\mathrm{K}: \rho\left(I_{n}\right) \rightarrow \rho\left(I_{n}\right)$ by

$$
\mathrm{K}(\mathrm{A})=\left\{\begin{array}{ll}
\text { Aif } I_{n}-\{i\} \subseteq & \text { A for some } \mathrm{i} \in I \\
\phi, & \text { otherwise }
\end{array},\right.
$$

then $\mu=\{\phi, X\} \cup\left\{A \subset I_{n}-\{\mathrm{i}\}, \mathrm{i}=1,2, \ldots, \mathrm{n}\right\}$, the co singleton generalized topology defined on a finite set. The only $\mu$-closed sets are $\phi, X$ and singleton subsets of $I_{n}$. In this space, the family of all $g_{\mu}$-closed sets and the family of all $\mu$ - closed sets coincide. For the topology $\tau=\{\phi\} \cup\{\mathrm{G} \subset X /\{1,2\} \subset \mathrm{G}\}$ on $X$, the $\mu$-closed sets are precisely the $g \mu$-closed sets. This space $(X, \tau)$ with the family of all generalized open sets $\mu$ which is strong, is also not $\mu \mathrm{g}$ - regular.

Example 3.6: Consider the space $(X, \tau)$ and generalized topology $\mu$ of the Example 2.3 of [9]. In this space, $\{\mathrm{a}, \mathrm{c}\}$ is $\mathrm{g} \mu$-closed but it is not $g_{\mu}^{*}-$ closed and also not $g_{\mu}$-closed. If $(X, \mu)$ is any generalized spaces which is not strong, then in [6, proposition 1.2], it is established that $X \in \sigma$ and so it follows that always $X \in \mathrm{b}$.And $X \in \beta$. The following example shows that in general, $X \notin \mu$, then $X \notin \lambda$ for $\lambda \in\{\mu, \delta, \alpha, \pi, \gamma, \eta\}$.

Example 3.7: Let $X$ be the set of all real numbers and $\mu=\{\phi\{0\}\}$. Then $X \notin$ $\lambda$ where $\lambda \in\{\mu, \delta, \alpha, \pi, \gamma, \eta\}$.

Lemma 3.10: Let $(X, \mu)$ be a GTS which is not strong. Then the following hold.

(i) $X \notin \pi$ and hence $X \notin \alpha$,

(ii) $X \notin \delta$ and hence $X \notin \eta$

Note that, if is a generalized topology on $X$, and let $M_{\mu}=\cup\{\mathrm{A} / A \in \mu\} . X$ $\notin \mu$ and $\lambda$ belongs to the family of all $\mu$ - open sets $\mu$, or the family of all $\alpha$ open sets $\alpha$, or the family of all Semi open sets $\sigma$ or the family of all pre open sets $\pi$ or the family of all $b$ - open sets $b$ or the family of all $\beta$ - open sets $\beta$. Then by the Lemma 3.10, we have $M_{\lambda} \neq X$ if $\in\{\mu, \alpha, \pi, \delta\}$ and $M_{\lambda}=\mathrm{X}$ if $\lambda \in\{\sigma, b, \beta, \varepsilon, \xi, \psi\}$ Moreover, $M_{\lambda}=M_{\mu}$ if $M_{\lambda} \neq X$.

Definition 3.10: Let $\mathrm{X}$ be a non-empty set and let $\mu$ be a generalized topology on $\mathrm{X}$. The space $(\mathrm{X}, \mu)$ is said to be $g \mu$-regular if for each pair consisting of a point $x \in M_{\lambda}$ and a $g_{\mu}^{*}$ - closed set F not containing $x$, there exist disjoint $\mu$ open sets $\mathrm{U}$ and $\mathrm{V}$ such that $x \in U$ and $\mathrm{F} \subset \mathrm{V}$.

Remark: Every $g \mu$-regular space is a $\mu$-regular space. 


\section{REFERENCES}

[1] Csaszar, A., Generalized topology, generalized continuity. (2002). Acta. Mathematica Hungarica, 96 (4), 351-357.

http://dx.doi.org/10.1023/A:1019713018007

[2] Csaszar, A., Extremally disconnected generalized topologies. (2004). Annales Univ. Budapest, Sectio Math, 17, 151-165.

[3] Csaszar, A., Generalized open sets in generalized topologies. (2005). Acta Mathematica Hungarica, 106, 1:2, 53-66.

[4] Csaszar, A., d and q modifications of generalized topologies. (2008). Acta Mathematica Hungarica, 120 (3), 275-279. http://dx.doi.org/10.1007/s10474-007-7136-9

[5] Dhana Balan, A.P., $\mu$-Continuous Functions on Generalized topology and certain Allied Structures. (2014). Math. Sci. Int. Research jou, 3 (1), 180-183.

[6] Guldurdek, A and Ozbakir, O.B., On - semi open sets. (2005). Acta Mathematica Hungarica, 109 (4), 347-355.

[7] Levine, N., A decomposition of continuity in topological spaces, (1961) Amer. Math Monthly 68, 44-66. http://dx.doi.org/10.2307/2311363

[8] Maragathavalli, S., Sheik John, M and Sivaraj, D., On g - closed sets in generalized topological spaces. (2010). J.Adv. Res. Pure. Maths. 2 (1), 57-64,

[9] Noiri, T, and Roy, B., Unification of generalized open sets on topological spaces. (2010). Acta. Math. Hungarica, 130 (4), 349 - 357.

[10] Roy, B, and Jafari, S., On covering properties via generalized open sets. (2002). Annales universitatis scientiarum Budapestinensis de Rolando Ectros Nominate Mathematica, 55, 57 - 65.

[11] Roy, B., On a type of generalized open sets. (2011). Applied General Topology, 12 (2), 163-173.
A Class of Separation Axioms in Generalized Topology 